\title{
Some subclasses of close-to-convex functions
}

\author{
by ADAM LECKO (Rzeszów)
}

\begin{abstract}
For $\alpha \in[0,1]$ and $\beta \in(-\pi / 2, \pi / 2)$ we introduce the classes $C_{\beta}(\alpha)$ defined as follows: a function $f$ regular in $U=\{z:|z|<1\}$ of the form $f(z)=z+\sum_{n=1}^{\infty} a_{n} z^{n}$, $z \in U$, belongs to the class $C_{\beta}(\alpha)$ if $\operatorname{Re}\left\{e^{i \beta}\left(1-\alpha^{2} z^{2}\right) f^{\prime}(z)\right\}>0$ for $z \in U$. Estimates of the coefficients, distortion theorems and other properties of functions in $C_{\beta}(\alpha)$ are examined.
\end{abstract}

1. Denote by $U=\{z \in \mathbb{C}:|z|<1\}$ the unit disk in the complex plane $\mathbb{C}$. Let $P$ denote the class of functions $p$ of the form $p(z)=1+p_{1} z+p_{2} z^{2}+\ldots$, $z \in U$, which are regular in $U$ and have a positive real part. Denote by $\Omega$ the class of functions $\omega$ regular in $U$ such that $\omega(0)=0$ and $|\omega(z)|<1$ for $z \in U$. A regular function $f$ in $U$ is called subordinate to a regular function $F$ in $U$ if there exists a function $\omega \in \Omega$ such that $f(z)=F(\omega(z)), z \in U$. We write then $f \prec F$ or $f(z) \prec F(z), z \in U$.

DEFinition 1.1. A function $f$ of the form

$$
f(z)=z+a_{2} z^{2}+\ldots+a_{n} z^{n}+\ldots, \quad z \in U,
$$

regular in $U$ belongs to the class $C_{\beta}(\alpha), \alpha \in \mathbb{C}, \beta \in(-\pi / 2, \pi / 2)$, if

$$
\operatorname{Re}\left\{e^{i \beta}\left(1-\alpha^{2} z^{2}\right) f^{\prime}(z)\right\}>0, \quad z \in U .
$$

We also set

$$
C(\alpha)=\bigcup_{\beta \in(-\pi / 2, \pi / 2)} C_{\beta}(\alpha)
$$

If $\alpha=|\alpha| e^{i \theta}, \theta \in[0,2 \pi)$, and $f \in C_{\beta}(\alpha), \beta \in(-\pi / 2, \pi / 2)$, then the function $g(z)=e^{-i \theta} f\left(e^{i \theta} z\right), z \in U$, belongs to $C_{\beta}(|\alpha|)$. Thus we may assume that $\alpha$ is real. By (1.2) it is sufficient to take $\alpha$ from the interval [0,1] because the assumption $|\alpha|>1$ implies that $C_{\beta}(\alpha)=\emptyset$ for all $\beta \in(-\pi / 2, \pi / 2)$.

1991 Mathematics Subject Classification: Primary 30C45.

Key words and phrases: close-to-convex functions, close-to-convex functions with argument $\beta$, functions convex in the direction of the imaginary axis, functions of bounded rotation with argument $\beta$. 
Hengartner and Schober [4] established that the inequality

$$
\operatorname{Re}\left\{\left(1-z^{2}\right) f^{\prime}(z)\right\}>0, \quad z \in U,
$$

characterizes the class of univalent functions $f$ of the form

$$
f(z)=a_{1} z+a_{2} z^{2}+\ldots+a_{n} z^{n}+\ldots, \quad a_{1} \in \mathbb{C},\left|a_{1}\right|=1, z \in U,
$$

with the normalization

$$
\liminf _{z \rightarrow-1} \operatorname{Re} f(z)=\inf _{z \in U} \operatorname{Re} f(z), \quad \limsup _{z \rightarrow 1} \operatorname{Re} f(z)=\sup _{z \in U} \operatorname{Re} f(z)
$$

which map $U$ onto domains convex in the direction of the imaginary axis. This class was denoted by $C \widetilde{V}_{2}(i)$. The condition (1.3) implies that Re $f^{\prime}(0)$ $=\operatorname{Re} a_{1}>0$.

Following the definition of $\alpha$-spiral functions (Špaček [10]) and functions close-to-convex with argument $\beta$ (Goodman and Saff [3]) we introduce in (1.3) the factor $e^{i \beta}=f^{\prime}(0)$. Therefore for $\beta \in(-\pi / 2, \pi / 2)$ we distinguish the class $\beta-C V_{2}(i)$ of functions $f$ of the form (1.1) regular in $U$ defined by the inequality

$$
\operatorname{Re}\left\{e^{i \beta}\left(1-z^{2}\right) f^{\prime}(z)\right\}>0, \quad z \in U .
$$

Thus for $\alpha=1$ and fixed $\beta \in(-\pi / 2, \pi / 2)$ we have $C_{\beta}(1)=\beta-C V_{2}(i)$.

Of course, if $f \in \beta-C V_{2}(i), \beta \in(-\pi / 2, \pi / 2)$, then the function $g(z)=$ $e^{i \beta} f(z), z \in U$, belongs to $C \widetilde{V}_{2}(i)$. Conversely, if $f \in C \widetilde{V}_{2}(i)$, then there exists $\beta \in(-\pi / 2, \pi / 2)$ such that the function $g(z)=e^{-i \beta} f(z), z \in U$, belongs to $\beta-C V_{2}(i)$.

For $\alpha=0,(1.2)$ yields a univalence condition found independently by Noshiro [9] and Warschawski [12]. The class of functions that satisfy this condition:

$$
\operatorname{Re}\left\{e^{i \beta} f^{\prime}(z)\right\}>0, \quad z \in U,
$$

is usually denoted by $P^{\prime}(\beta)$ and the functions are called of bounded rotation with argument $\beta$.

Notice that (1.2) can be written as

$$
\operatorname{Re}\left\{\alpha^{2} e^{i \beta}\left(1-z^{2}\right) f^{\prime}(z)+\left(1-\alpha^{2}\right) e^{i \beta} f^{\prime}(z)\right\}>0 .
$$

Taking $\gamma=\alpha^{2}, \alpha \in[0,1]$, we see that the left hand side of (1.2) is a convex combination of the left hand sides of (1.4) and (1.5). This method of defining new classes of analytic functions is due to Mocanu [8] who introduced the $\alpha$-convex functions. This concept was used by many authors. For example, in [1] the classes $H(\alpha)$, with $\alpha$ real, of functions $f$ of the form (1.1) regular in $U$ are defined by the inequality

$$
\operatorname{Re}\left\{(1-\alpha) f^{\prime}(z)+\alpha\left(1+z \frac{f^{\prime \prime}(z)}{f^{\prime}(z)}\right)\right\}>0, \quad z \in U .
$$

The class $C_{0}(\alpha)$ was examined in $[6]$. 
2. In this section estimates of the coefficients of functions in $C_{\beta}(\alpha)$ are obtained.

Theorem 2.1. If $f \in C_{\beta}(\alpha), \alpha \in[0,1], \beta \in(-\pi / 2, \pi / 2)$, then $f$ is univalent in $U$.

Proof. For $\alpha=0$ this is shown in [9] and [12].

Let now $\alpha \in(0,1]$. The function

$$
\varphi_{\alpha}(z)=\frac{1}{2 \alpha} \log \frac{1+\alpha z}{1-\alpha z}, \quad z \in U, \quad \varphi_{\alpha}(0)=0,
$$

is convex and univalent in $U$. Moreover, if $f \in C_{\beta}(\alpha)$, where $\beta \in(-\pi / 2, \pi / 2)$, then

$$
\operatorname{Re}\left\{e^{i \beta} \frac{f^{\prime}(z)}{\varphi_{\alpha}^{\prime}(z)}\right\}=\operatorname{Re}\left\{e^{i \beta}\left(1-\alpha^{2} z^{2}\right) f^{\prime}(z)\right\}>0, \quad z \in U .
$$

This means that $f$ is close-to-convex and univalent (see [5]).

Theorem 2.2. If $\beta \in(-\pi / 2, \pi / 2), \alpha_{1}, \alpha_{2} \in[0,1]$ and $\alpha_{1} \neq \alpha_{2}$, then $C_{\beta}\left(\alpha_{1}\right) \nsubseteq C_{\beta}\left(\alpha_{2}\right)$ and $C_{\beta}\left(\alpha_{2}\right) \nsubseteq C_{\beta}\left(\alpha_{1}\right)$.

Proof. Let $0 \leq \alpha_{2}<\alpha_{1} \leq 1$.

$1^{\circ}$. Let $f$ be the solution of the equation

$$
e^{i \beta}\left(1-\alpha_{1}^{2} z^{2}\right) f^{\prime}(z)=\frac{1+z^{2}}{1-z^{2}} \cos \beta+i \sin \beta, \quad z \in U,
$$

where $\beta \in(-\pi / 2, \pi / 2)$. Of course, $f \in C_{\beta}\left(\alpha_{1}\right)$ and by (2.1) we have

$$
\begin{gathered}
\operatorname{Arg}\left\{e^{i \beta}\left(1-\alpha_{2}^{2} z^{2}\right) f^{\prime}(z)\right\}=\operatorname{Arg}\left\{\left(\frac{1+z^{2}}{1-z^{2}} \cos \beta+i \sin \beta\right) \frac{1-\alpha_{2}^{2} z^{2}}{1-\alpha_{1}^{2} z^{2}}\right\} \\
=\operatorname{Arg}\left\{\frac{1+z^{2}}{1-z^{2}} \cos \beta+i \sin \beta\right\}+\operatorname{Arg} \frac{1-\alpha_{2}^{2} z^{2}}{1-\alpha_{1}^{2} z^{2}}
\end{gathered}
$$

where $z \in U, \operatorname{Arg}\left(e^{i \beta}\right)=\beta$ and $\operatorname{Arg} 1=0$.

Let now $z=e^{i t}, t \in(0, \pi) \cup(\pi, 2 \pi)$. Then

$$
\frac{1+z^{2}}{1-z^{2}}=i \frac{\cos t}{\sin t}
$$

and

$$
\frac{1+z^{2}}{1-z^{2}} \cos \beta+i \sin \beta=i\left(\frac{\cos t}{\sin t} \cos \beta+\sin \beta\right) .
$$

For fixed $\beta \in(-\pi / 2, \pi / 2)$ we can choose $t_{0} \in(0, \pi / 2)$ such that

$$
\frac{\cos t_{0}}{\sin t_{0}} \cos \beta+\sin \beta>0 \text {. }
$$


Set $z_{0}=e^{i t_{0}}$. From (2.3) and (2.4) we have

$$
\operatorname{Arg}\left\{\frac{1+z_{0}^{2}}{1-z_{0}^{2}} \cos \beta+i \sin \beta\right\}=\frac{\pi}{2} .
$$

On the other hand, if $z=e^{i t}$, where $t \in(0, \pi) \cup(\pi, 2 \pi)$, then

$$
\frac{1-\alpha_{2}^{2} z^{2}}{1-\alpha_{1}^{2} z^{2}}=\frac{1+\alpha_{1}^{2} \alpha_{2}^{2}-\left(\alpha_{1}^{2}+\alpha_{2}^{2}\right) \cos 2 t}{1-2 \alpha_{1}^{2} \cos 2 t+\alpha_{1}^{4}}+i \frac{\left(\alpha_{1}^{2}-\alpha_{2}^{2}\right) \sin 2 t}{1-2 \alpha_{1}^{2} \cos 2 t+\alpha_{1}^{4}} .
$$

The real part in (2.6) is positive for all $t \in(0, \pi) \cup(\pi, 2 \pi)$. Moreover, if $\alpha_{2}<\alpha_{1}$ and $t \in(0, \pi / 2)$, then the imaginary part in (2.6) is also positive. In particular, this holds for $t_{0}$. Therefore (2.6) yields

$$
0<\operatorname{Arg} \frac{1-\alpha_{2}^{2} z_{0}^{2}}{1-\alpha_{1}^{2} z_{0}^{2}}<\frac{\pi}{2} .
$$

Using (2.5) and (2.7) we conclude that

$$
\frac{\pi}{2}<\operatorname{Arg}\left\{\frac{1+z_{0}^{2}}{1-z_{0}^{2}} \cos \beta+i \sin \beta\right\}+\operatorname{Arg} \frac{1-\alpha_{2}^{2} z_{0}^{2}}{1-\alpha_{1}^{2} z_{0}^{2}}<\pi .
$$

Let now $\left(z_{n}\right), n \in \mathbb{N}$, where $z_{n}=r_{n} e^{i t_{0}}, 0<r_{n}<1$, be a sequence that converges to $z_{0}$. Then there is an $n_{0} \in \mathbb{N}$ such that for all $n>n_{0}$ inequalities (2.8) are satisfied with $z_{n}$ in place of $z_{0}$. Finally, by (2.2) and (2.8) for $n>n_{0}$ we have

$$
\frac{\pi}{2}<\operatorname{Arg}\left\{e^{i \beta}\left(1-\alpha_{2}^{2} z_{n}^{2}\right) f^{\prime}\left(z_{n}\right)\right\}<\pi .
$$

This means that $f \notin C_{\beta}\left(\alpha_{2}\right)$.

$2^{\circ}$. Let now $f$ be the solution of the equation

$$
e^{i \beta}\left(1-\alpha_{2}^{2} z^{2}\right) f^{\prime}(z)=\frac{1-z^{2}}{1+z^{2}} \cos \beta+i \sin \beta, \quad z \in U .
$$

Obviously, $f \in C_{\beta}\left(\alpha_{2}\right)$.

If $z=e^{i t}, t \in(0, \pi / 2) \cup(\pi / 2,3 \pi / 2) \cup(3 \pi / 2,2 \pi)$, then

$$
\frac{1-z^{2}}{1+z^{2}} \cos \beta+i \sin \beta=i\left(-\frac{\sin t}{\cos t} \cos \beta+\sin \beta\right) \text {. }
$$

For fixed $\beta \in(-\pi / 2, \pi / 2)$ we can choose $t_{0} \in(0, \pi / 2)$ such that

$$
-\frac{\sin t}{\cos t} \cos \beta+\sin \beta<0 .
$$

If we set $z_{0}=e^{i t_{0}}$, then from the above and (2.9) we have

$$
\operatorname{Arg}\left\{\frac{1-z_{0}^{2}}{1+z_{0}^{2}} \cos \beta+i \sin \beta\right\}=-\frac{\pi}{2} .
$$


For $\alpha_{2}<\alpha_{1}$ and $t=t_{0}$ the imaginary part in (2.6) is negative with $\alpha_{2}$ in place of $\alpha_{1}$ and vice versa. Therefore

$$
-\pi<\operatorname{Arg} \frac{1-\alpha_{1}^{2} z_{0}^{2}}{1-\alpha_{2}^{2} z_{0}^{2}}<-\frac{\pi}{2} .
$$

Hence and from (2.10) we conclude that

$$
-\pi<\operatorname{Arg}\left\{\frac{1-z_{0}^{2}}{1+z_{0}^{2}} \cos \beta+i \sin \beta\right\}+\operatorname{Arg} \frac{1-\alpha_{1}^{2} z_{0}^{2}}{1-\alpha_{2}^{2} z_{0}^{2}}<-\frac{\pi}{2} .
$$

Thus for $z \in U$ near to $z_{0}$ we have

$$
-\pi<\operatorname{Arg}\left\{e^{i \beta}\left(1-\alpha_{1}^{2} z^{2}\right) f^{\prime}(z)\right\}<-\frac{\pi}{2}
$$

This means that $f \notin C_{\beta}\left(\alpha_{1}\right)$ and ends the proof.

Now we find coefficient bounds for the class $C_{\beta}(\alpha)$.

TheOREM 2.3. If $f \in C_{\beta}(\alpha), \alpha \in(0,1), \beta \in(-\pi / 2, \pi / 2)$ and $f$ is of the form (1.1), then, for all $k \in \mathbb{N}$,

$$
\begin{gathered}
\left|a_{2 k}\right| \leq \frac{1-\alpha^{2 k}}{\left(1-\alpha^{2}\right) k} \cos \beta \\
\left|a_{2 k+1}\right| \leq \frac{2 \cos \beta+(1-2 \cos \beta) \alpha^{2 k}-\alpha^{2(k+1)}}{\left(1-\alpha^{2}\right)(2 k+1)} .
\end{gathered}
$$

Proof. By (1.2) there exists a function

$$
q(z)=\cos \beta+i \sin \beta+\sum_{n=1}^{\infty} q_{n} z^{n}, \quad z \in U,
$$

such that $\operatorname{Re} q(z)>0$ for $z \in U$ and

$$
e^{i \beta}\left(1-\alpha^{2} z^{2}\right) f^{\prime}(z)=q(z)
$$

Then for $\beta \in(-\pi / 2, \pi / 2)$ the function

$$
p(z)=\frac{1}{\cos \beta}(q(z)-i \sin \beta)=1+p_{1} z+p_{2} z^{2}+\ldots+p_{n} z^{n}+\ldots, \quad z \in U,
$$

belongs to $P$. Moreover,

$$
q_{n}=p_{n} \cos \beta, \quad n \in \mathbb{N} .
$$

Equating coefficients in (2.13) we have

$$
\begin{gathered}
2 e^{i \beta} a_{2}=q_{1}, \quad e^{i \beta}\left(3 a_{3}-\alpha^{2}\right)=q_{2}, \quad \ldots, \\
e^{i \beta}\left[(n+1) a_{n+1}-(n-1) \alpha^{2} a_{n-1}\right]=q_{n} .
\end{gathered}
$$

It follows from (2.14) and (2.15) that

$$
a_{n+1}=\frac{(n-1) \alpha^{2} a_{n-1}+e^{-i \beta} p_{n} \cos \beta}{n+1} .
$$


If $n=2 k-1, k \in \mathbb{N}$, then from (2.16) we have

$$
a_{2 k}=\frac{e^{-i \beta} \cos \beta}{2 k} \sum_{j=1}^{k} \alpha^{2(k-j)} p_{2 j-1} .
$$

Hence using the known estimates $\left|p_{n}\right| \leq 2, n \in \mathbb{N}$, we obtain

$$
\left|a_{2 k}\right| \leq \frac{\cos \beta}{k} \sum_{j=1}^{k} \alpha^{2(k-j)}=\frac{1-\alpha^{2 k}}{\left(1-\alpha^{2}\right) k} \cos \beta .
$$

If $n=2 k, k \in \mathbb{N}$, then (2.16) yields

$$
a_{2 k+1}=\frac{\alpha^{2 k}+e^{-i \beta} \cos \beta \sum_{j=1}^{k} \alpha^{2(k-j)} p_{2 j}}{2 k+1} .
$$

Hence

$$
\left|a_{2 k+1}\right| \leq \frac{\alpha^{2 k}+2 \cos \beta \sum_{j=1}^{k} \alpha^{2(k-j)}}{2 k+1}=\frac{2 \cos \beta+(1-2 \cos \beta) \alpha^{2 k}-\alpha^{2(k+1)}}{\left(1-\alpha^{2}\right)(2 k+1)},
$$

for $k \in \mathbb{N}$. This ends the proof of the theorem.

The bound in (2.11) is sharp and achieved by the function $f_{\alpha, \beta}, \alpha \in$ $(0,1), \beta \in(-\pi / 2, \pi / 2)$, which is the solution of the differential equation

i.e.

$$
e^{i \beta}\left(1-\alpha^{2} z^{2}\right) f_{\alpha, \beta}^{\prime}(z)=\frac{1+z}{1-z} \cos \beta+i \sin \beta,
$$

$$
\begin{aligned}
f_{\alpha, \beta}(z)= & e^{-i \beta}\left\{\frac{\cos \beta}{1-\alpha^{2}}\left(\log \frac{1-\alpha^{2} z^{2}}{(1-z)^{2}}-\frac{1+\alpha^{2}}{2 \alpha} \log \frac{1+\alpha z}{1-\alpha z}\right)\right. \\
& \left.+i \sin \beta \frac{1}{2 \alpha} \log \frac{1+\alpha z}{1-\alpha z}\right\}, \quad z \in U .
\end{aligned}
$$

For the third coefficient $a_{3}$ we get the sharp bound

$$
\left|a_{3}\right| \leq \frac{2 \cos \beta+\alpha^{2}}{3} .
$$

Equality is attained when $p_{2}=2 e^{i \beta}$ in (2.18). This gives the extremal function $g_{\alpha, \beta}, \alpha \in(0,1), \beta \in(-\pi / 2, \pi / 2)$, which is the solution of the equation

i.e.

$$
e^{i \beta}\left(1-\alpha^{2} z^{2}\right) g_{\alpha, \beta}^{\prime}(z)=\frac{1+e^{i \beta / 2} z}{1-e^{i \beta / 2} z} \cos \beta+i \sin \beta,
$$

$$
\begin{aligned}
g_{\alpha, \beta}(z)= & \frac{e^{-i \beta} \cos \beta}{2 \alpha\left(\alpha^{2}-e^{i \beta}\right)}\left[4 \alpha e^{i \beta / 2} \log \left(1-e^{i \beta / 2} z\right)\right. \\
& \left.+\left(\alpha-e^{i \beta / 2}\right)^{2} \log (1+\alpha z)-\left(\alpha+e^{i \beta / 2}\right)^{2} \log (1-\alpha z)\right] \\
& +i e^{-i \beta} \sin \beta \frac{1}{2 \alpha} \log \frac{1+\alpha z}{1-\alpha z}, \quad z \in U
\end{aligned}
$$


It is not known if the bounds for odd-numbered coefficients $a_{n}, n \geq 5$, of functions $f \in C_{\beta}(\alpha)$, for $\beta \neq 0$, are sharp. If $\beta=0$, then the estimates are sharp and are the same as in Corollary 2.4 below.

Corollary 2.4. If $f \in C(\alpha), \alpha \in(0,1)$, and $f$ is of the form (1.1), then

$$
\left|a_{2 k}\right| \leq \frac{1-\alpha^{2 k}}{\left(1-\alpha^{2}\right) k} \quad \text { and } \quad\left|a_{2 k+1}\right| \leq \frac{2-\alpha^{2 k}-\alpha^{2(k+1)}}{\left(1-\alpha^{2}\right)(2 k+1)}, \quad k \in \mathbb{N} .
$$

The above results are sharp. The function

$f_{\alpha, 0}(z)=\frac{1}{1-\alpha^{2}}\left(\log \frac{1-\alpha^{2} z^{2}}{(1-z)^{2}}-\frac{1+\alpha^{2}}{2 \alpha} \log \frac{1+\alpha z}{1-\alpha z}\right), \quad z \in U, \alpha \in(0,1)$, is extremal for all coefficients.

Observe that the formulas (2.16), (2.17) and (2.18) for the coefficients also hold for $\alpha=0$ and $\alpha=1$. Therefore we can also obtain estimates in these two cases. For $\alpha=0$, from (2.16) we have

$$
a_{n}=\frac{e^{-i \beta} p_{n-1} \cos \beta}{n}, \quad n \in \mathbb{N} .
$$

This formula gives the well known result:

Corollary 2.5. If $f \in P^{\prime}(\beta), \beta \in(-\pi / 2, \pi / 2)$, and $f$ is of the form (1.1), then

$$
\left|a_{n}\right| \leq \frac{2}{n} \cos \beta, \quad n \in \mathbb{N} .
$$

In particular, for $\beta=0$,

$$
\left|a_{n}\right| \leq \frac{2}{n}, \quad n \in \mathbb{N}
$$

(see [7]).

The estimates (2.20) and (2.21) can be obtained from (2.11) and (2.12) by putting $\alpha=0$. The following functions are extremal for the classes $P^{\prime}(\beta)$ and $P^{\prime}(0)$, respectively:

$$
\begin{aligned}
& f_{0, \beta}(z)=\lim _{\alpha \rightarrow 0} f_{\alpha, \beta}(z)=e^{-i \beta}\left[-e^{-i \beta} z-2 \cos \beta \log (1-z)\right], \quad z \in U, \\
& f_{0,0}(z)=\lim _{\alpha \rightarrow 0} f_{\alpha, 0}(z)=-z-2 \log (1-z), \quad z \in U .
\end{aligned}
$$

Moreover, inequalities (2.21) are satisfied in the class $C(0)$ and equality holds for $f_{0,0}$. The bounds $(2.21)$ can be obtained from (2.19) by putting $\alpha=0$.

For $\alpha=1$, from (2.17) and (2.18) we have

$$
a_{2 k}=\frac{e^{-i \beta} \cos \beta}{2 k} \sum_{j=1}^{k} p_{2 j-1}, \quad a_{2 k+1}=\frac{1+e^{-i \beta} \cos \beta \sum_{j=1}^{k} p_{2 j}}{2 k+1}, \quad k \in \mathbb{N} \text {. }
$$


These two formulas yield the following result due to Hengartner and Schober (see [4], Theorem 3):

Corollary 2.6. If $f \in \beta-C V_{2}(i), \beta \in(-\pi / 2, \pi / 2)$, and $f$ is of the form (1.1), then

$$
\begin{aligned}
\left|a_{2 k}\right| & \leq \cos \beta \\
\left|a_{2 k+1}\right| & \leq \frac{2 k \cos \beta+1}{2 k+1}, \quad k \in \mathbb{N} .
\end{aligned}
$$

In particular, for $\beta=0$,

$$
\left|a_{n}\right| \leq 1, \quad n \in \mathbb{N} .
$$

The function

$$
f_{1, \beta}(z)=\lim _{\alpha \rightarrow 1} f_{\alpha, \beta}(z)=e^{-i \beta}\left[\frac{z}{1-z} \cos \beta+\frac{i \sin \beta}{2} \log \frac{1+z}{1-z}\right],
$$

$\beta \in(-\pi / 2, \pi / 2), z \in U$, makes (2.22) sharp. On the other hand, if $\beta \neq 0$, then (2.23) is sharp only for $k=1$ and for the function

$$
\begin{aligned}
g_{1, \beta}(z) & =\frac{e^{-i \beta} \cos \beta}{2\left(1-e^{i \beta}\right)}\left[4 e^{i \beta / 2} \log \left(1-e^{i \beta / 2} z\right)\right. \\
& \left.+\left(1-e^{i \beta / 2}\right)^{2} \log (1+z)-\left(1+e^{i \beta / 2}\right)^{2} \log (1-z)\right]+\frac{i e^{-i \beta}}{2} \sin \beta \log \frac{1+z}{1-z},
\end{aligned}
$$$$
z \in U \text { (see [4]). }
$$

If $\beta=0$, then (2.24) is sharp and equality is achieved by the function

$$
f_{1,0}(z)=\lim _{\alpha \rightarrow 1} f_{\alpha, 0}(z)=\frac{z}{1-z}, \quad z \in U .
$$

Moreover, the estimates (2.24) hold for the class $C(1)$ and $f_{1,0}$ is extremal in this case.

3. Now we give some distortion theorems for the class $C_{\beta}(\alpha)$.

TheOREM 3.1. If $f \in C_{\beta}(\alpha), \alpha \in[0,1], \beta \in(-\pi / 2, \pi / 2)$, then

$$
\begin{aligned}
& \left|f^{\prime}(z)\right| \leq \frac{\sqrt{1+r^{4}+2 r^{2} \cos 2 \beta}+2 r \cos \beta}{\left(1-\alpha^{2} r^{2}\right)\left(1-r^{2}\right)}=\frac{\exp \left(\operatorname{arsh} \frac{2 r \cos \beta}{1-r^{2}}\right)}{1-\alpha^{2} r^{2}} \\
& \left|f^{\prime}(z)\right| \geq \frac{\sqrt{1+r^{4}+2 r^{2} \cos 2 \beta}-2 r \cos \beta}{\left(1+\alpha^{2} r^{2}\right)\left(1-r^{2}\right)}=\frac{\exp \left(-\operatorname{ar} \operatorname{sh} \frac{2 r \cos \beta}{1-r^{2}}\right)}{1+\alpha^{2} r^{2}}
\end{aligned}
$$

and

$$
|f(z)| \leq \int_{0}^{r} \frac{\sqrt{1+\varrho^{4}+2 \varrho^{2} \cos 2 \beta}+2 \varrho \cos \beta}{\left(1-\alpha^{2} \varrho^{2}\right)\left(1-\varrho^{2}\right)} d \varrho,
$$




$$
|f(z)| \geq \int_{0}^{r} \frac{\sqrt{1+\varrho^{4}+2 \varrho^{2} \cos 2 \beta}-2 \varrho \cos \beta}{\left(1+\alpha^{2} \varrho^{2}\right)\left(1-\varrho^{2}\right)} d \varrho
$$

for $z \in U,|z| \leq r<1$.

Proof. By Lemma 5 of [4] equation (1.2) may be written as

$$
\left(1-\alpha^{2} z^{2}\right) f^{\prime}(z)=\frac{1+e^{-2 i \beta} \omega(z)}{1-\omega(z)}, \quad z \in U,
$$

where $\omega \in \Omega$. Thus

$$
f^{\prime}(z)=\frac{1}{1-\alpha^{2} z^{2}} \frac{1+e^{-2 i \beta} \omega(z)}{1-\omega(z)} .
$$

Moreover, we have

$$
\frac{1+e^{-2 i \beta} \omega(z)}{1-\omega(z)} \prec \frac{1+e^{-2 i \beta} z}{1-z}, \quad z \in U .
$$

In view of (3.6) and by Theorem 2.3 of [11],

$$
\begin{aligned}
\frac{\left|1+e^{-2 i \beta} r^{2}\right|-\left|1+e^{-2 i \beta}\right| r}{1-r^{2}} & \leq\left|\frac{1+e^{-2 i \beta} \omega(z)}{1-\omega(z)}\right| \\
& \leq \frac{\left|1+e^{-2 i \beta} r^{2}\right|+\left|1+e^{-2 i \beta}\right| r}{1-r^{2}},
\end{aligned}
$$

where $z \in U,|z| \leq r<1$. Now, the upper and lower bounds (3.1) and (3.2) follow from (3.5) and (3.7).

The estimates (3.7) are sharp and in view of (3.6) are realized by the function

$$
p_{0}(z)=\frac{1+e^{-2 i \beta} z}{1-z}, \quad z \in U,
$$

at two points $z_{0}$ and $z_{1}$ of modulus $r$. Let $z_{0}=r e^{i \theta_{0}(\beta)}$ and $z_{1}=r e^{i \theta_{1}(\beta)}$, where $0<r<1, \theta_{0}(\beta), \theta_{1}(\beta) \in[0,2 \pi)$, give the lower and upper bound in (3.7) respectively. Now, we denote by $h_{\alpha, \beta}, \alpha \in[0,1], \beta \in(-\pi / 2, \pi / 2)$, the function which is the solution of the equation (3.5) for $\omega=\omega_{0}$ defined by

$$
\omega_{0}(z)=-i e^{i \theta_{0}(\beta)} z, \quad z \in U .
$$

The function $h_{\alpha, \beta}$ is extremal for the lower estimate (3.2) and equality is attained at the point $z=i r$.

In the same way we denote by $t_{\alpha, \beta}, \alpha \in[0,1], \beta \in(-\pi / 2, \pi / 2)$, the function which is the solution of the equation (3.5) for $\omega=\omega_{1}$ given by

$$
\omega_{1}(z)=e^{i \theta_{1}(\beta)} z, \quad z \in U .
$$

Then $t_{\alpha, \beta}$ gives the maximum modulus in (3.1) at the point $z=r$ and is extremal for the upper estimate.

Now we show the estimates (3.3) and (3.4). 
For $z \in U,|z|=r$, the upper bound (3.3) follows immediately from (3.1). Let now $\xi \in U,|\xi|=r$, be a point such that $|f(\xi)|=\min \{|f(z)|:|z|=r\}$. Moreover, let $I=[0, f(\xi)]$ denote the closed line segment from 0 to $f(\xi)$. Thus for $|z|=r$ we have

$$
\begin{aligned}
|f(z)| & \geq|f(\xi)|=\int_{I}|d w|=\int_{f^{-1}(I)}\left|f^{\prime}(z)\right||d z| \\
& \geq \int_{0}^{r} \frac{\sqrt{1+\varrho^{4}+2 \varrho^{2} \cos 2 \beta}-2 \varrho \cos \beta}{\left(1+\alpha^{2} \varrho^{2}\right)\left(1-\varrho^{2}\right)} d \varrho .
\end{aligned}
$$

The estimates $(3.3),(3.4)$ are sharp and realized by the functions $h_{\alpha, \beta}$ and $t_{\alpha, \beta}$.

Corollary 3.2. If $f \in C(\alpha), \alpha \in(0,1)$, then

$$
\begin{gathered}
\frac{1-r}{(1+r)\left(1+\alpha^{2} r^{2}\right)} \leq\left|f^{\prime}(z)\right| \leq \frac{1+r}{(1-r)\left(1-\alpha^{2} r^{2}\right)}, \\
\frac{1}{1+\alpha^{2}}\left[\log \frac{(1+r)^{2}}{1+\alpha^{2} r^{2}}-\left(1-\alpha^{2}\right) \frac{1}{\alpha} \arctan (\alpha r)\right] \\
\leq|f(z)| \leq \frac{1}{1-\alpha^{2}}\left[\log \frac{1-\alpha^{2} r^{2}}{(1-r)^{2}}-\frac{1+\alpha^{2}}{2 \alpha} \log \frac{1+\alpha r}{1-\alpha r}\right],
\end{gathered}
$$

where $z \in U,|z|=r<1$.

The estimates (3.8) and (3.9) are sharp. The upper and lower bounds are achieved when $\beta=0$. In this case $\theta_{1}(0)=0, \theta_{0}(0)=\pi$ and, respectively, $\omega_{1}(z)=z, \omega_{0}(z)=i z$. The extremal functions $h_{\alpha, 0}$ and $t_{\alpha, 0}$ have the following form:

$$
\begin{aligned}
h_{\alpha, 0}(z)=f_{\alpha, 0}(z)=\frac{1}{1-\alpha^{2}}\left(\log \frac{1-\alpha^{2} z^{2}}{(1-z)^{2}}-\frac{1+\alpha^{2}}{2 \alpha} \log \frac{1+\alpha z}{1-\alpha z}\right), \quad z \in U, \\
t_{\alpha, 0}(z)=\frac{i}{1+\alpha^{2}}\left(2 \log (1-i z)+\frac{1}{2 \alpha i}(\alpha-i)^{2} \log (1+\alpha z)\right. \\
\left.\quad-\frac{1}{2 \alpha i}(\alpha+i)^{2} \log (1-\alpha z)\right), \quad z \in U .
\end{aligned}
$$

The function $t_{\alpha, 0}$ can be rewritten as

$$
\begin{aligned}
t_{\alpha, 0}(z) & =\frac{i}{1+\alpha^{2}}\left(2 \log (1-i z)-\log \left(1-\alpha^{2} z^{2}\right)-\frac{1-\alpha^{2}}{2 i \alpha} \log \frac{1+\alpha z}{1-\alpha z}\right) \\
& =\frac{i}{1+\alpha^{2}}\left(\log \frac{(1-i z)^{2}}{1-\alpha^{2} z^{2}}+\left(1-\alpha^{2}\right) \frac{1}{\alpha} \arctan (\alpha i z)\right) .
\end{aligned}
$$

Putting $\alpha=\beta=0$ in (3.1)-(3.4) we obtain known results (see [7]): 
Corollary 3.3. If $f \in P^{\prime}(0)$, then

$$
\begin{aligned}
\frac{1-r}{1+r} & \leq\left|f^{\prime}(z)\right| \leq \frac{1+r}{1-r}, \\
2 \log (1+r)-r & \leq|f(z)| \leq-2 \log (1-r)-r
\end{aligned}
$$

for $z \in U,|z|=r<1$.

The functions

$$
h_{0,0}(z)=-z-2 \log (1-z), \quad z \in U,
$$

and

$$
t_{0,0}(z)=\lim _{\alpha \rightarrow 1} t_{\alpha, 0}(z)=i \log (1-i z)^{2}-z, \quad z \in U,
$$

are respective extremal functions for the upper and lower bounds.

The next corollary is obtained from Theorem 3.1 by putting $\alpha=0$ and $\beta=1$ (see [4]).

Corollary 3.4. If $f \in C_{0}(1)$, then

$$
\begin{gathered}
\frac{1-r}{(1+r)\left(1+r^{2}\right)} \leq\left|f^{\prime}(z)\right| \leq \frac{1}{(1-r)^{2}}, \\
\frac{1}{2} \log \frac{(1+r)^{2}}{1+r^{2}} \leq|f(z)| \leq \frac{r}{1-r}
\end{gathered}
$$

for $z \in U,|z|=r<1$.

The functions

$$
h_{1,0}(z)=\frac{z}{1-z}, \quad z \in U, \quad \text { and } \quad t_{1,0}(z)=\frac{i}{2} \log \frac{(1-i z)^{2}}{1-z^{2}}, \quad z \in U,
$$

are extremal.

In the limit cases as $\alpha$ tends to 0 or to 1 , the bounds (3.8) and (3.9) give sharp results for the classes $C(0)$ and $C(1)$ that agree with (3.10), (3.11) and with (3.12), (3.13) respectively.

The lower bound in (3.9) yields

Corollary 3.5. If $f \in C(\alpha), \alpha \in(0,1]$, then $f(U)$ contains the disk

$$
|w|<\frac{1}{1+\alpha^{2}}\left[\log \frac{4}{1+\alpha^{2}}-\left(1-\alpha^{2}\right) \frac{1}{\alpha} \arctan \alpha\right]
$$

(see [6]).

The constant on the right hand side of (3.14) is best possible and the function $t_{\alpha, 0}$ is extremal.

For the class $C(0)$ the following result is known (see [2]):

Corollary 3.6. If $f \in C(0)$, then $f(U)$ contains the disk

$$
|w|<2 \log 2-1 \text {. }
$$


This constant can be obtained from (3.14) by letting $\alpha \rightarrow 0$.

If $\alpha=1$, then Corollary 3.5 reduces to the result obtained by Hengartner and Schober [4]:

COROLlary 3.7. If $f \in C(1)$, then $f(U)$ contains the disk

$$
|w|<\frac{1}{2} \log 2 \text {. }
$$

\section{References}

[1] H. S. Al-Amiri and M. O. Reade, On a linear combination of some expressions in the theory of univalent functions, Monatsh. Math. 80 (4) (1975), 257-264.

[2] I. M. Gal'perin, The theory of univalent functions with bounded rotation, Izv. Vyssh. Ucheb. Zaved. Mat. 1958 (3) (4), 50-61 (in Russian).

[3] A. W. Goodman and E. B. Saff, On the definition of a close-to-convex function, Internat. J. Math. and Math. Sci. 1 (1978), 125-132.

[4] W. Hengartner and G. Schober, On schlicht mappings to domains convex in one direction, Comment. Math. Helv. 45 (1970), 303-314.

[5] W. Kaplan, Close-to-convex schlicht functions, Michigan Math. J. 1 (1952), 169185.

[6] A. Lecko, On some classes of close-to-convex functions, Fol. Sci. Univ. Tech. Resov. 60 (1989), 61-70.

[7] T. H. MacGregor, Functions whose derivative has a positive real part, Trans. Amer. Math. Soc. 104 (1962), 532-537.

[8] P. T. Mocanu, Une propriété de convexité généralisée dans la théorie de la représentation conforme, Mathematica (Cluj) 11 (34) (1969), 127-133.

[9] K. Noshiro, On the theory of schlicht functions, J. Fac. Sci. Hokkaido Univ. Jap. (1) 2 (1934-1935), 129-155.

[10] L. Špaček, Contribution à la theorie des fonctions univalentes, Časopis Pěst. Mat. 2 (1932), 12-19.

[11] J. Stankiewicz and J. Waniurski, Some classes of functions subordinate to linear transformation and their applications, Ann. Univ. Mariae Curie-Skłodowska Sect. A 28 (1974), 85-94.

[12] S. Warschawski, On the higher derivatives at the boundary in conformal mapping, Trans. Amer. Math. Soc. 38 (1935), 310-340.

DEPARTMENT OF MATHEMATICS

TECHNICAL UNIVERSITY OF RZESZÓW

W. POLA 2

35-959 RZESZÓW, POLAND 